\title{
Adsorption of water in silica nanopores: Impacts of Surface Hydrophilicity and Pore Size
}

\author{
GERNOT ROTHER $^{1 *}$, ANDREW STACK ${ }^{1}$, DAVID COLE ${ }^{2}$, \\ TINGTING LIU ${ }^{2}$, SIDDHARTH GAUTAM ${ }^{2}$, ANDREAS \\ $\mathrm{BUSCH}^{3}$
}

${ }^{1}$ Chemical Sciences Division, Oak Ridge National

Laboratory, rotherg@ornl.gov, stackag@ornl.gov

${ }^{2}$ Ohio State University, cole.618@osu.edu,

liu.2189@osu.edu, gautam.25@osu.edu

${ }^{3}$ Heriot Watt University, h.busch@hw.ac.uk

Water uptake at $298 \mathrm{~K}$ by two mesoporous MCM-41 silicas with different pore sizes was studied using volumetric vapor sorption. Through variation of sample pretreatment temperature and time, the number of surface hydroxyl groups was varied, leading to marked changes in the water sorption behavior. With increasing surface hydroxyl density, the adsorption of water to the pore surfaces increases, leading to formation of a thicker and denser water sorption layer. The BET model was used to measure surface hydroxyl density from low pressure parts of the water adsorption isotherms. Adsorption of water molecules scales with the number density of surface hydroxyl groups, and monolayer coverage is reached at reduced pressure of ca. 0.3. The onset of the adsorption pore condensation of water shifts to lower reduced pressure with increasing surface hydroxyl density, indicating growing thickness of adsorption layers. However, the water pore condensation step of the desorption branch shifts to smaller reduced pressure with increasing surface hydroxylation, reducing the adsorption-desorption hysteresis width. An adsorbed phase model is utilized to calculate the distribution of pore water molecules between adsorbed and pore condensation phases, and characterize the density and thickness of the water sorption phase. In the smaller pores, the adsorbed phase forms a sparse monolayer, while approximately a bilayer is formed in the wider pores. The previously reported silica nanopore underfilling by water is confirmed and rationalized by a significantly reduced water density in the adsorbed phase with respect to bulk, while the pore core is filled with water of approximately bulk density. 